\title{
Phase diagram of the G(2) Higgs model and G(2)-QCD
}

\author{
Björn H. Wellegehausen* \\ Theoretisch-Physikalisches Institut, University Jena \\ E-mail: bjoern.wellegehausen@uni-jena.de
}

\begin{abstract}
We investigate gauge theories based on the smallest exceptional simple lie group $G_{2}$. Our first model considered here is $G_{2}$ Yang-Mills coupled to a fundamental Higgs field. In 4 spacetime dimensions we explore the phase diagram of the theory, showing that at larger Higgs masses the first order deconfinement phase transition turns into a crossover and therefore connects the low temperature confined phase with the high temperature deconfined phase. The second model investigated is $G_{2}$ Yang-Mills coupled to fundamental fermions. It shares many features with QCD, especially fermionic baryons, but due to the absence of the fermion sign problem we can investigate this theory with Monte Carlo techniques even at low temperature and high baryonic density. First results on small lattices are presented.
\end{abstract}

XXIX International Symposium on Lattice Field Theory

July 10-16 2011

Squaw Valley, Lake Tahoe, California

${ }^{*}$ Speaker. 


\section{Introduction}

Due to the fermion sign problem of QCD at real quark chemical potential, Monte Carlo techniques are not directly applicable to investigate the QCD phase diagram, especially at low temperatures and high densities [1]. Understanding the theory in this region of the QCD parameter space is very important for dense quark systems and the formation of compact stellar objects. Currently, the only reliable information is obtained from continuum methods, which usually require approximations or QCD-like theories having as many features as possible in common with QCD. One example of those theories is two colour two flavour QCD. Its phase diagram was explored in a series of papers $[2,3]$. The main drawback of two colour QCD is the absence of fermionic degrees of freedom in the hadron spectrum. In this work we investigate QCD-like theories based on the exceptional gauge group $G_{2}$. It was already shown that $G_{2}$ Yang Mills theory undergoes a first order phase transition from the low temperature confined phase to the high temperature deconfined phase [4,5]. Additionally, the gauge group $S U(3)$ of strong interaction is a subgroup of $G_{2}$ and this observation has interesting consequences [6]. With a Higgs field in the fundamental 7-dimensional representation one can break the $G_{2}$ gauge symmetry to the $S U(3)$ symmetry via the Higgs mechanism. When the Higgs field in the action

$$
S[A, \phi]=\int d^{4} x\left(\frac{1}{4 g^{2}} \operatorname{tr} F_{\mu v} F^{\mu v}+\frac{1}{2}\left(D_{\mu} \phi, D_{\mu} \phi\right)+V(\phi)\right)
$$

picks up a vacuum expectation value $v$, the 8 gluons belonging to $S U(3)$ remain massless and the additional 6 gauge bosons acquire a mass proportional to $v$. In the limit $v \rightarrow \infty$ they are removed from the spectrum such that $G_{2}$ Yang-Mills-Higgs (YMH) theory reduces to $S U$ (3) Yang-Mills theory. Even more interesting, for intermediate and large values of $v$, the $G_{2} \mathrm{YMH}$-theory mimics $S U$ (3) gauge theory with dynamical 'scalar quarks'. The second theory presented in this work is $G_{2}$-QCD [6] with the action given by

$$
S[A, \psi]=\int d^{4} x\left(\frac{1}{4 g^{2}} \operatorname{tr} F_{\mu v} F^{\mu v}+\frac{1}{2} \bar{\psi}\left(\gamma_{\mu} D^{\mu}+m+\gamma_{0} \mu\right) \psi\right) .
$$

Here $\psi$ is a massive Dirac spinor in the fundamental 7-dimensional representation of $G_{2}$. We show that even at finite quark chemical potential $\mu$ the fermion determinant is non-negative. This allows us to investigate the phase diagram at zero temperature and finite chemical potential. Moreover the spectrum contains fermionic baryons and we expect the theory to behave in many aspects very similar to QCD.

\section{The confinement-deconfinement transition in $G_{2}$ Yang-Mills}

For a gauge group with non-trivial center the trace of the Polyakov loop in representation $\mathscr{R}$

$$
P_{\mathscr{R}}(\vec{x})=\operatorname{tr}_{\mathscr{R}} \mathscr{P}(\vec{x}), \quad \mathscr{P}(\vec{x})=\frac{1}{N_{c}} \operatorname{tr}\left(\operatorname{exp~i} \int_{0}^{\beta_{T}} A_{0}(\tau, \vec{x}) d \tau\right), \quad \beta_{T}=\frac{1}{T},
$$

tranforms under center transformations like $P_{\mathscr{R}}(\vec{x}) \mapsto z^{k} P_{\mathscr{R}}(\vec{x})$ where $\mathrm{z}$ is an element of the center of the gauge group and $k$ is the $\mathrm{N}$-ality of the representation $\mathscr{R}$. In a pure gauge theory the only 

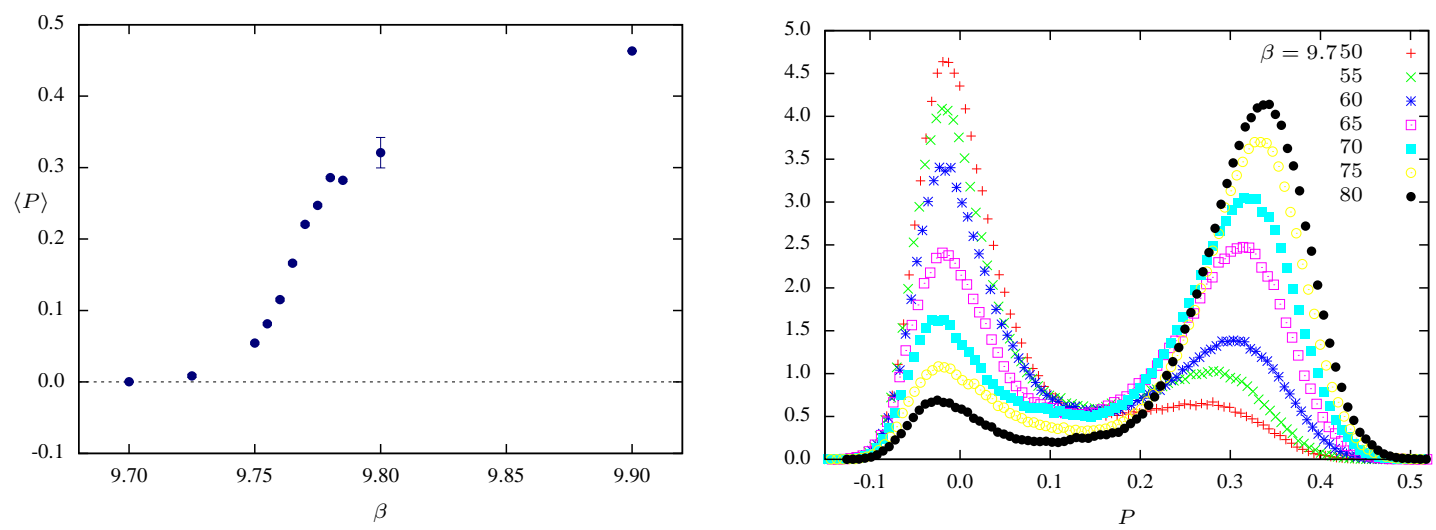

Figure 1: Left panel: Polyakov loop expectation values at the finite temperature confinement-deconfinement transition on a $16^{3} \times 6$ lattice. Right panel: Histograms of the Polyakov loop in the vicinity of the phase transition point $\beta_{\text {crit }}=9.765$, pointing to a first order deconfinement transition.

dynamical degrees of freedom are gluons in the adjoint center-blind representation. Therefore the Polyakov loop in any representation with non-zero $\mathrm{N}$-ality is an order parameter for the spontaneous breaking of center symmetry. On the other hand the vacuum expectation value of the Polyakov loop is related to the free energy of an infinitely heavy test quark in the gluonic bath, $\langle P\rangle \propto e^{-\beta F}$. Consequently, in the confined phase center symmetry is unbroken, while it is spontaneously broken in the deconfined phase. If we couple fundamental matter fields, as for example in QCD, the center symmetry is explicitly broken and the Polyakov loop is not an order parameter for confinement anymore. But it is still expected that QCD confines colour in the sense that charges of test quarks are screened by dynamical light quarks. In $G_{2}$ gluodynamics the situation is very similar to the QCD case: Quarks and anti-quarks transform under the fundamental 7-dimensional representation. Since the center of $G_{2}$ is trivial these fundamental charges can be screened by at least three gluons in the adjoint 14-dimensional representation,

$$
(7) \otimes(14) \otimes(14) \otimes(14)=(1) \oplus \cdots .
$$

The Polyakov loop ceases to be an order parameter for confinement. In both, $G_{2}$ gluodynamics and QCD, confinement can be identified with a linear rising inter-quark potential at intermediate scales and string breaking at larger distances in every representation of the gauge group [7, 8]. Nevertheless on a finite lattice the Polyakov loop serves as an approximate order parameter which changes rapidly at the phase transition and is small (but non-zero) in the confining phase. We see a clear signal in the Polyakov loop at the confinement-deconfinement transition and the double peak structure in the histograms points to a first order confinement-deconfinement transition for $G_{2}$ gluodynamics (Fig. 1), which is in agreement with earlier results in [4, 5].

\section{The $G_{2}$ Higgs model}

The lattice action for the $G_{2}$ Yang-Mills-Higgs theory (1.1) reads

$$
S_{\mathrm{YMH}}[\mathscr{U}, \Phi]=\beta \sum_{\square}\left(1-\frac{1}{7} \operatorname{tr} \operatorname{Re} \mathscr{U} \square\right)-\kappa \sum_{x \mu} \Phi_{x+\hat{\mu}} \mathscr{U}_{x, \mu} \Phi_{x}, \quad \Phi_{x} \cdot \Phi_{x}=1,
$$


where $\Phi$ is a seven component normalized real scalar field. In the limit $\beta \rightarrow \infty$ the gauge bosons, belonging to the coset space $G_{2} / S U(3) \sim S O(7) / S O(6) \sim S^{6}$, decouple and the theory reduces to an $S O(7)$-invariant nonlinear $\sigma$-model. It shows spontaneous symmetry breaking down to $S O(6)$ at a second order phase transition. With respect to the $S U$ (3) subgroup of $G_{2}$ the fundamental representations (7) and (14) branch into the following irreducible $S U(3)$-representations:

$$
(7) \longrightarrow(3) \oplus(\overline{3}) \oplus(1) \quad, \quad(14) \longrightarrow(8) \oplus(3) \oplus(\overline{3}) .
$$

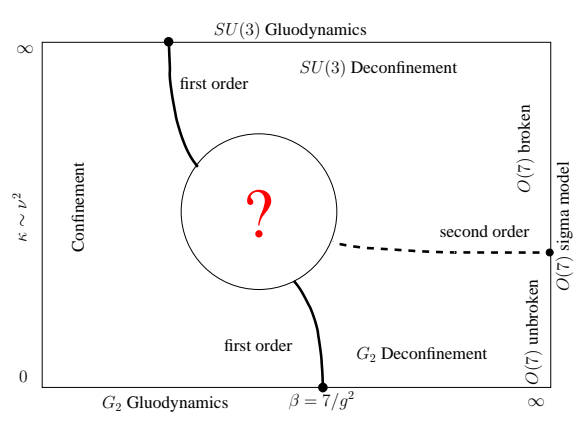

Figure 2: Expected phase diagram in the parameter space $\left(7 / g^{2}, \kappa\right)$.

The Higgs field branches into a scalar quark, scalar antiquark and singlet with respect to $S U(3)$. Similarly, a $G_{2}$-gluon branches into a massless $S U(3)$-gluon and additional gauge bosons with respect to $S U(3)$. The latter eat up the non-singlet scalar fields such that the spectrum in the broken phase consists of 8 massless gluons, 6 massive gauge bosons and one massive Higgs particle. In the limit of $\kappa \rightarrow \infty$ these 6 massive gauge bosons become infinitely heavy and the theory reduces to $S U(3)$ Yang Mills theory. If $\kappa$ is lowered, in addition to the 8 gluons of $S U(3)$, the 6 additional gauge bosons of $G_{2}$ begin to participate in the dynamics. Similarly as dynamical quarks and anit-quarks in QCD, they transform in the representations (3) and ( $\overline{3})$ of $S U(3)$ and thus explicitly break the $\mathbb{Z}_{3}$ center symmetry. As in QCD they are expected to weaken the deconfinement phase transition [4]. For $\kappa=0$ we recover $G_{2}$ gluodynamics with a first order deconfinement phase transition. Fig. 2 shows the expected phase diagram. Our results for the complete phase diagram in the $(\beta, \kappa)$ plane as calculated on $16^{3} \times 6$ up to $24^{3} \times 6$ lattices are summarized in Fig. 3. We calculated histograms and susceptibilities of the Polyakov loop, the plaquette density and the Higgs action density near the marked points on the transition lines in this figure. From earlier calculations on smaller lattices we suggest that a triple point exists. An extrapolation to the point where the confined phase meets both deconfined phases leads to the couplings $\beta_{\text {trip }}=9.62(1)$ and $\kappa_{\text {trip }}=1.455(5)$. Near this point the deconfinement transition is very weak, continuous or absent. Therefore we performed high-statistics simulations to investigate this region in parameter space more carefully. Up to a rather small region surrounding $\left(\beta_{\text {trip }}, \kappa_{\text {trip }}\right)$ we can show that the deconfinement transition is first order and the Higgs transition is second order. But in this small window in parameter space, around the would-be triple point, the deconfinement transition is either second order or absent [9].

\section{4. $G_{2}-\mathbf{Q C D}$}

The lattice action for $G_{2}-\mathrm{QCD}(1.2)$ reads

$$
S_{\mathrm{QCD}}[\mathscr{U}, \Psi]=\beta\left(S_{\mathrm{Sym}}[\mathscr{U}]-\frac{1}{2} \sum_{x y} \bar{\Psi}_{x} D_{x y}[\mathscr{U}, m, \mu] \Psi_{y}\right),
$$

where $S_{\text {Sym }}$ is the Symanzik improved gauge action and $D[\mathscr{U}, m, \mu]$ the Wilson-Dirac operator at bare fermion mass $m$ and real quark chemical potential $\mu$. If a unitary operator $T$ exists such 

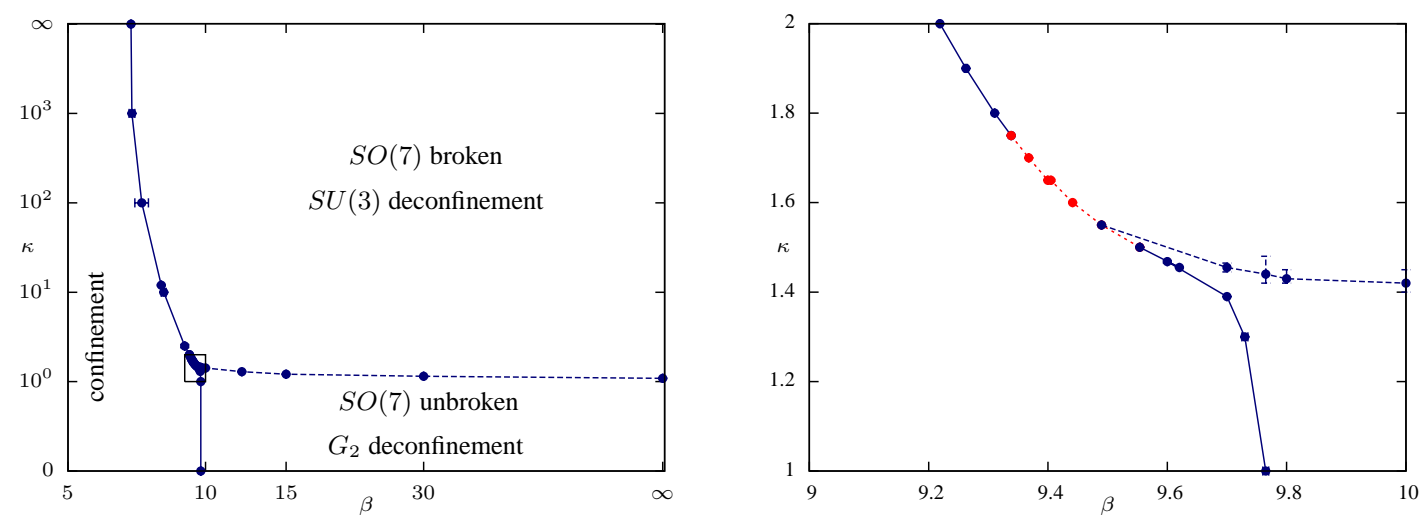

Figure 3: Phase transition lines on a $16^{3} \times 6$ lattice. The solid line corresponds to the first order deconfinement transitions and the dashed line to the second order Higgs transitions. The plot on the right panel shows the details inside the small box in the plot on the left panel where the transition lines almost meet. The dotted line between the first order lines corresponds to a window where the transition is a crossover.

that the Dirac operator obeys the relation $D^{*} T=T D$ with $T^{*} T=-\mathbb{1}$ and $T^{\dagger}=T^{-1}$, the fermion determinant is non-negative [2]. For Wilson fermions this implies the conditions $T \mathscr{U} T^{\dagger}=\mathscr{U}^{*}$ and $T \gamma_{\mu} T^{\dagger}=\gamma_{\mu}^{*}$. Since every representation of $G_{2}$ is real, we immediately find (for an Euclidean representation of the $\gamma$-matrices and charge conjugation matrix $C$ )

$$
T=\mathbb{1} \otimes C \gamma_{5} \quad \Rightarrow \quad T^{*} T=-\mathbb{1} \quad \Rightarrow \quad \operatorname{det} D[\mathscr{U}, m, \mu] \geq 0 .
$$

This feature of the fermion determinant makes Monte Carlo techniques applicable and allows us to investigate the complete phase diagram of $G_{2}$-QCD on the lattice. The chiral symmetry of $N_{f}$ flavor QCD is $S U\left(N_{f}\right)_{L} \otimes S U\left(N_{f}\right)_{R} \otimes U(1)_{B}$. Since the quark representation for $G_{2}$ is real the dirac spinor decomposes into two Majorana spinors for vanishing mass. Consequently, we find an additional flavour symmetry and the continuous $U(1)_{B}$ turns into the discrete group $\mathbb{Z}(2)$. The chiral symmetry of $G_{2}-\mathrm{QCD}$ is then [6]

$$
S U\left(2 N_{f}\right)_{L=R^{*}} \otimes \mathbb{Z}(2)_{B}
$$

Due to the discrete baryon number symmetry we can only distinguish between states with an even or odd number of quarks. Similar to QCD, mesons consist of an even number of quarks. Baryons are fermionic bound states with an odd number of quarks, for example, three quarks or a single quark and three gluons. Our first simulations are performed on rather small lattices with a spatial extend of $L=8$ and $T=2$ for finite temperature and on a $8^{4}$ lattice in the case of zero temperature. The observables considered in this preliminary study are the Polyakov loop $P$, the chiral condensate $\chi=\frac{1}{V} \frac{\partial \ln Z}{\partial m}$ and the quark number density $n_{\mathrm{q}}=\frac{1}{V} \frac{\partial \ln Z}{\partial \mu}$. First we compare the finite temperature phase transition at zero chemical potential to the phase transition in pure gauge theory. Our results for the Polyakov loop and the chiral condensate are shown in Fig. 4 (left panel). If we increase the hopping parameter from $\kappa=0$ to $\kappa=0.147$, the transition in the Polyakov loop becomes weaker and the critical temperature decreases. This behaviour is also seen in the chiral condensate. The difference between its value in the chirally symmetric and broken phase grows with decreasing mass, due to the smaller explicit breaking of chiral symmetry. In Fig. 4 (right panel) we show the 

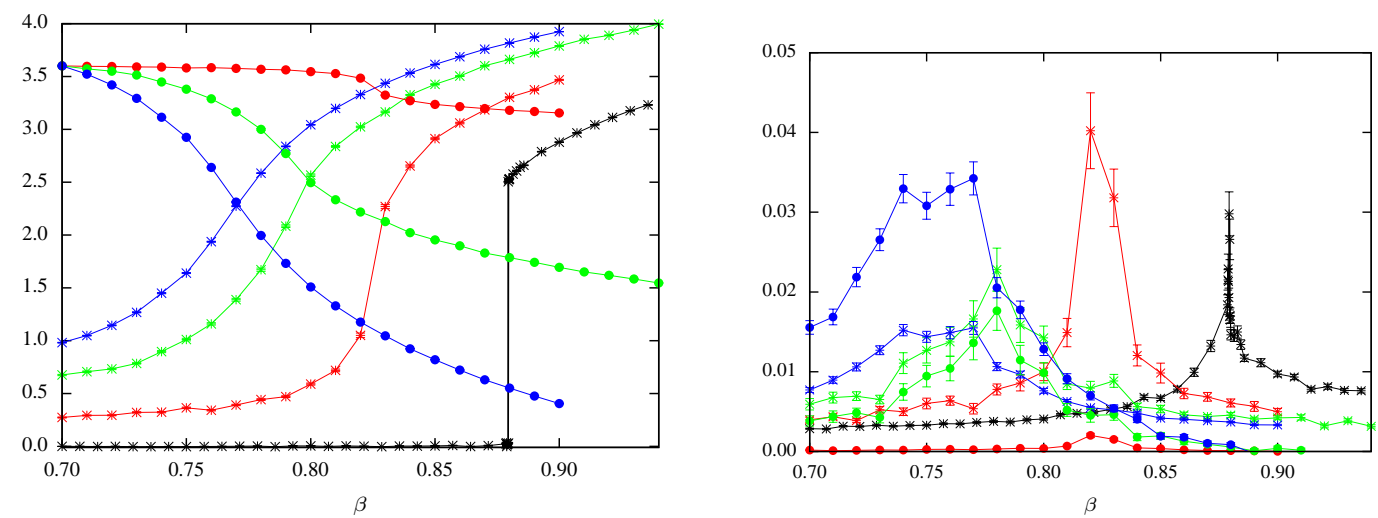

Figure 4: Left panel: Polyakov loop (stars) and (rescaled) chiral condensate (dots) at finite temperature and zero chemical potential for $\kappa=0,0.096,0.131,0.147$. Right panel: Susceptibility of the Polyakov loop and chiral condensate.

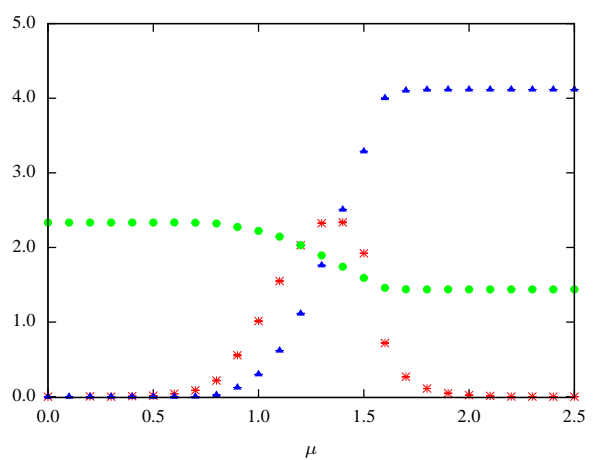

Figure 5: Polyakov loop (stars), chiral condensate (dots) and quark number density (triangles) at zero temperature. susceptibilites of both quantities. The peak becomes very broad for smaller quark masses, pointing to a crossover. On these rather small lattices and within the given resolution in temperature, we can see no difference in the position of the peak. But whether the deconfinement and chiral transition critical temperature coincide and whether the transition really is a crossover at smaller quark masses has to be investigated in further simulations on larger lattices. At zero temperature the system stays in the vacuum until at approximately $\mu=0.8$ the quark number density increases, Fig. 5 . At $\mu \approx 1.5$ the system is saturated in the sence that each lattice site is occupied by an odd number of quarks. We also observe a transition in the Polyakov loop together with a drop in the chiral condensate. This points to a phase transition, or a crossover, from a confined phase to a chirally symmetric deconfined phase. At larger values of the chemical potential the Polyakov loop decreases again. This may be an lattice artifact due to the saturation of the system and has to be investigated further. If we increase the temperature again, the transition becomes weaker as shown in Fig. 6.
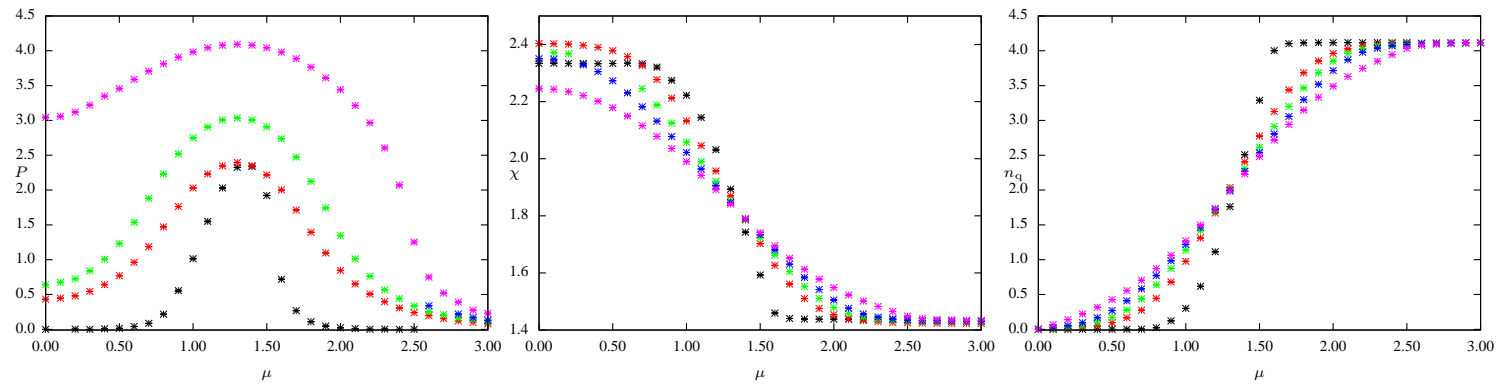

Figure 6: Polyakov loop $P$ (left panel), chiral condensate $\chi$ (center panel) and quark number density $n_{\mathrm{q}}$ (right panel) for different temperatures. 


\section{Conclusions}

We have investigated QCD-like theories based on the gauge group $G_{2}$. If we couple $G_{2}$ Yang-Mills to a fundamental Higgs field, the theory mimics, in the broken phase, QCD with scalar quarks and anti-quarks with respect to the $S U(3)$ subgroup of $G_{2}$. In the limit of infinitely heavy quarks we recover $S U(3)$ gauge theory with a first order deconfinement phase transition. If we decrease the mass the transition becomes weaker and, in a very small window in parameter space, turns into a crossover. This behaviour is already very similar to QCD. The second theory investigated is one flavour $G_{2}$-QCD which has many features in common with QCD. In this first study we show that the finite temperature phase transition becomes weaker with decreasing mass of the quarks. At zero temperature and finite chemical potential we find a transition in the Polyakov loop and the chiral condensate together with an increasing baryon number density. First results on larger lattices will be published in a forthcoming paper [10].

\section{Acknowledgments}

The author thanks Andreas Wipf, Axel Maas and Christian Wozar for collaboration and support. Helpful discussions with Lorenz von Smekal, Christof Gattringer, Kurt Langfeld, Uwe-Jens Wiese and Štefan Olejník are gratefully acknowledged. This work has been supported by the DFG under GRK 1523. The simulations in this paper were carried out at the Omega-Cluster of the TPI.

\section{References}

[1] C. Gattringer, C.B. Lang, Quantum chromodynamics on the lattice, Lect. Notes Phys. (2010)

[2] S. Hands, I. Montvay, S. Morrison, M. Oevers, L. Scorzato, J. Skullerud, Numerical study of dense adjoint matter in two color QCD, Eur. Phys. J. C17 (2000) 285

[3] S. Hands, S. Kim, and J. Skullerud, A Quarkyonic Phase in Dense Two Color Matter?, Phys. Rev. D81 (2010) 091502

[4] M. Pepe and U. J. Wiese, Exceptional Deconfinement in G(2) Gauge Theory, Nucl. Phys. B768 (2007) 21

[5] G. Cossu, M. D’Elia, A. Di Giacamo, B. Lucini, C. Pica, $G_{2}$ gauge theory at finite temperature, JHEP 10 (2007) 100

[6] K. Holland, P. Minkowski, M. Pepe and U. J. Wiese, Exceptional confinement in G(2) gauge theory, Nucl. Phys. B668 (2003) 207

[7] J. Greensite, K. Langfeld, Š. Olejník, H. Reinhardt and T. Tok, Color screening, Casimir scaling, and domain structure in $G(2)$ and $S U(N)$ gauge theories, Phys. Rev. D75 (2007) 034501

[8] B. H. Wellegehausen, A. Wipf and C. Wozar, Casimir Scaling and String Breaking in G(2) Gluodynamics, Phys. Rev. D83 (2011) 016001

[9] B. H. Wellegehausen, A. Wipf and C. Wozar, Phase diagram of the lattice G(2) Higgs Model, Phys. Rev. D83 (2011) 114502

[10] B. H. Wellegehausen, A. Maas, L.v. Smekal and A. Wipf, Lattice simulations of a QCD-like theory with fermionic baryons at finite baryon density, in preparation 